\title{
Modeling pulsed laser micromachining of micro geometries using machine-learning techniques
}

\author{
Teixidor, D., Grzenda M., Bustillo, A., Ciurana, J. \\ Department of Mechanical Engineering and Industrial Construction \\ Universitat de Girona, Girona, Spain \\ Faculty of Mathematics and Information Science, Warsaw University of Technology, \\ Warsaw, Poland \\ Department of Civil Engineering, University of Burgos, Burgos, Spain
}

\begin{abstract}
A wide range of opportunities are emerging in the micro-system technology sector for laser micro-machining systems, because they are capable of processing a various types of materials with micro-scale precision. However, few process datasets and machine learning techniques are optimized for this industrial task. This article aims to show how the process parameters of micro-laser milling influence the final features of the microshapes that are produced and aims to identify, at the same time, the most accurate machine learning technique for the modelization of this multivariable process. We studied the capabilities of laser micromachining by performing experiments on hardened steel with a pulsed Nd:YAG laser. Arrays of micro-channels were manufactured using various scanning speeds, pulse intensities and pulse frequencies. The results are presented in terms of the main industrial requirements for any manufactured good: dimensional accuracy (in our case, depth and width of the channels), surface roughness and material removal rate (which is a measure of the productivity of the process). Different machine learning techniques were then tested on the datasets to try to build high accuracy models for each output variable. The selected techniques were: k-Nearest Neighbours, neural networks, decision trees and linear regression models. Our analysis of the correlation coefficients and the mean absolute error of all the generated models show that neural networks are better at modelling channel width and that decision trees are better at modelling surface roughness; both techniques are similar for depth and material removal rate. In all cases these two techniques are more accurate than the other two. It can be concluded that decision trees can be used for modelling laser micro-machining of micro geometries, if the dimensional accuracy of the workpiece is the main industrial requirement, while neural networks are better in the other cases.
\end{abstract}




\section{Introduction}

Laser systems are increasingly employed in many diverse micro-system technology sectors such as biomedicine, automotive manufacture, telecommunications, display devices, printing technologies and semiconductors (Rizvi, 2002). Material removal during the laser machining process depends, to a certain degree, on the characteristics of the laser and the properties of the workpiece; however, it is primarily affected by the interaction between the laser and the workpiece (Pham, 2007). In real factory conditions, this interaction is influenced by other types of machine-tool parameters that are easily controlled, such as pulse frequency, peak power, scanning speed and overlapping. Although many of these process parameters can be adjusted, in order to obtain the desired quality and to optimize the efficiency of the features being produced, there is a lack of knowledge about how they affect the laser machining process, especially in new sensitive applications like micro-machining of the shape of micro geometries (Brousseau, 2011).

Various studies have investigated how laser process parameters affect the quality of the resultant machined features. Campanelli et al. (Campanelli, 2007) analyzed the influence of frequency, scanning strategy and overlap on depth and surface roughness, during laser machining of an aluminum-magnesium alloy. The experiments and the analysis of variance showed that, in general, optimizing surface roughness was conversely related to maximizing depth. Cicala et al. (Cicala, 2008) studied the effects of pulse frequency, power, scanning speed and overlap on the MRR and surface roughness. The results showed that pulse frequency and scanning speed were the main parameters affecting surface roughness, which was reduced with lower scanning speeds and higher frequencies. The Material Removal Rate (MRR) mainly depends on pulse frequency alone. Bartolo et al. (Bartolo, 2006) analyzed the incidence of the same parameters while looking at the scanning strategy, in the process of machining channels in tempered steel and aluminum. Their results suggested that, with lower frequencies and reduced laser power, the surface quality is better. However, both parameters need to be increased, in order to achieve an optimum value for a higher MRR. Kaldos et al. (Kaldos, 2004) used a CNC milling machine with a Nd:YAG laser source, on die steel, to study the impact of lamp current, pulse frequency, overlapping and scanning speed on surface roughness and the MRR. They concluded that an increase in current intensity or an insufficient overlap of laser passes results in a less well finished surface. Semaltianos et al. (Semaltianos, 2010) studied the effects of fluence and pulse frequency on surface roughness and MRR in nickel-based alloys with a Nd:YVO4 picosecond laser. They also analyzed the surface morphology of these alloys with AFM and SEM techniques.

Ciurana et al. (Ciurana, 2009) used a pulsed Nd:YAG laser to study the effect of the process parameters on minimum volume error and surface roughness in laser machined tool steel for macro scale geometry, although micro scale geometry was not evaluated. The experimental results were inconsistent for large shapes. Dhara et al. (Dhara, 2008) micro-machined die steel while modifying pulse intensity, pulse frequency, pulse duration and air pressure, in order to predict the optimum process parameter settings for maximum depth with a minimum recast 
layer. Kumar et al. (Kumar, 2010) investigated the influence of laser power, pulse frequency, number of scans and air pressure, on the groove depth in the generation of micro-notches with a nanosecond pulsed fiber laser on stainless steel and aluminum. Karazi et al. (Karazi, 2009) machined and characterized micro-channel formation by laser machining. They studied the effects of laser power, pulse frequency and scanning speed on the width and depth of the channels. They also modeled the process using Artificial Neural Networks (ANN).

The application of Artificial Intelligence (AI) techniques to model micro machining of metal components is an open issue. Most of the very few works on this topic focus on the application of ANNs to this task: the work of Desai et al. (Desai, 2012) predicted the depth of cut for singlepass laser micro-milling process using ANN and genetic programming approaches and the work of Karazi et al. (Karazi, 2009) compared ANN and DoE models for the prediction of lasermachined micro-channel dimensions. If we open the state of the art to the application of Al techniques to machining processes similar to laser milling, we can conclude that ANNs are the most common technique used for most of these processes such as milling, drilling or laser finishing (Chandrasekaran, 2010), although many other Al techniques have also been applied for such purposes. Bustillo et al. proposed the use of Bayesian Networks and ensembles to predict surface roughness in drilling (Bustillo, 2012), laser finishing (Bustillo 2011a) and roughing (Bustillo 2011b) operations, Grzenda et al. proposed different evolutionary algorithms to improve ANNs accuracy (Grzenda 2012a) and 2012b) in drilling and milling operations and Mahdavinejad et al. proposed the use of artificial immune systems to model milling processes (Mahdavinejad, 2012). In any case, most of the most recent works use ANNs as a standard technique to be improved by new approaches, such as those proposed by Bustillo et al. (Bustillo, 2011b), Correa et al. (Correa, 2009), Desai et al. (Desai, 2012), Mahdavinejad et al. (Mahdavinejadm 2012) and Diez-Pastor et al. (Diez-Pastor, 2012).

The aim of this work is to describe the information needed to improve the laser micromachining process in the production of microshapes and to develop a suitable AI model for the modelization of this industrial task. The process parameter settings are optimized with regression models developed from experimental work, to achieve the required dimensional precision, surface quality and productivity. Arrays of micro-channels are fabricated on hardened tool steel using laser machining processes, while measuring feature size, geometric accuracy, surface roughness and the MRR. This work will contribute to the selection of appropriate process parameters through an analysis of the influence of scanning speed, pulse frequency and pulse intensity on the final quality of the machined micro-feature. Moreover, machine learning methods are used to evaluate the complexity of prediction tasks. Representatives of rule-based, instance-based and linear and nonlinear models are applied. Prediction accuracy remains at different levels depending on the parameter to be modeled rather than the technique used to model it. Hence, the complexity of modeling individual features of interest has been determined. 


\section{Experimental set up}

The experiments, set up to study the influence of the process parameters, were carried out with a pulsed Nd:YAG, Deckel Maho Lasertec 40 machine, with 100W average laser power and a wavelength of $1,064 \mathrm{~nm}$.

Although the pulse intensity level on the surface was not measured during our experiments, based on the technical data of the laser system, we can provide an ideal pulse intensity level, which is given by:

$\mathrm{PI}=\frac{\mathrm{P}}{\pi\left(\frac{\mathrm{d}}{2}\right)^{2}}$

where, $P$ is the laser power $(100 \mathrm{~W})$, and $d$ is the beam spot diameter $(0.003 \mathrm{~cm})$. Therefore the ideal pulse intensity was estimated to be $1.4 \mathrm{~W} / \mathrm{cm} 2$. Furthermore, we can determine the ideal Peak Pulse Power (PPP), which is given by:

$\mathrm{PPP}=\frac{\mathrm{P}}{\tau}$

where, $\mathrm{P}$ is the laser power (100 W) and $\tau$ is the laser pulse duration (10 ns). For the laser characteristics used in this study, the PPP is estimated to be $10 \mathrm{MW} / \mathrm{s}$. The specifications of the

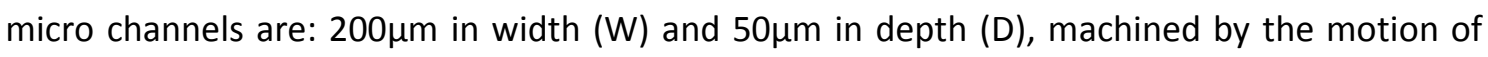
the laser beam in the $x$ and $y$ directions removing material in all three directions ( $x, y$ and $z)$. As shown in Figure 1, in order to machine the entire cavity, there is overlap between adjacent pulses (Oy) within a pass of length (L) and overlap between successive passes (Ox). All the experiments were performed with a laser spot size $(\varnothing)$ of $0.03 \mathrm{~mm}$ and a track displacement (distance between passes, a) of $10 \mu \mathrm{m}$. The overlap Ox between successive passes is given by (Samant, 2010):

$\mathrm{O}_{\mathrm{x}}=\left(1-\frac{\mathrm{a}}{\emptyset}\right) \cdot 100$

In this study, Ox was $66.6 \%$. The overlap between adjacent pulses (Oy) depends on the scanning speed, the pulse frequency and the spot diameter. It is therefore different for each experiment. Oy is given by:

$\mathrm{O}_{\mathrm{y}}=\left(1-\frac{\mathrm{SS}}{\mathrm{PF} \cdot \varnothing}\right) \cdot 100$

where, SS is the scanning speed and PF is the pulse frequency, which are different for each experiment.

The workpiece material was hardened AISI H13 tool steel, selected because it is a widely used material in the moulds and dies industry. 
Dimensional measurements were performed with a ZEISS SteREO Discovery.V12 stereomicroscope. Quartz PCIC software was used to measure the feature dimensions and Mitutoyo SV2000 Surftest equipment was used to measure surface roughness.

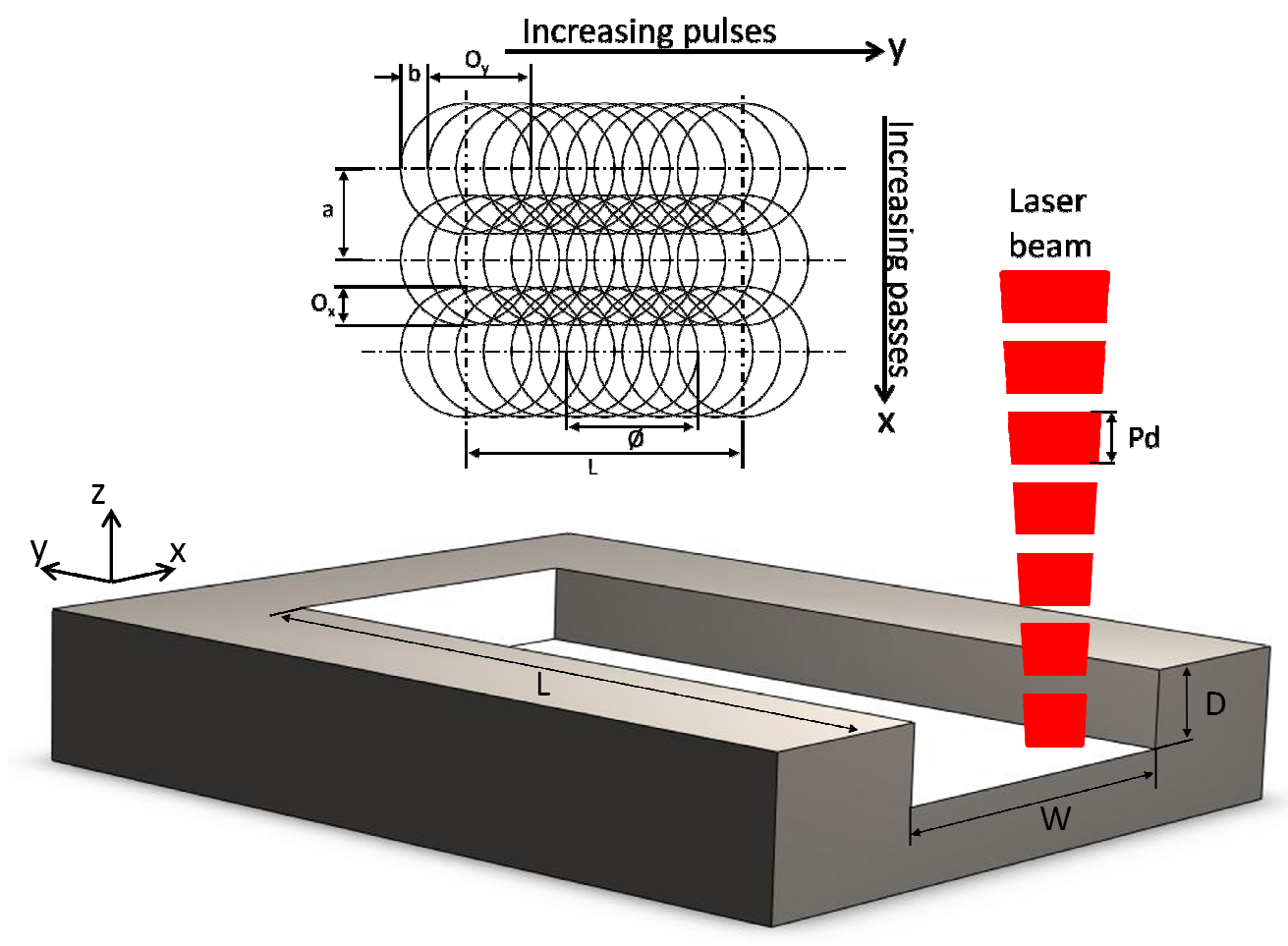

Figure 1. A schematic illustration of the 3D-laser milling and the overlapping of the laser pulses.

Some screening experiments were performed to select the appropriate factor levels of the process parameters. Several micro-channels were machined, in each case changing one single parameter while the others remained fixed. In this way, we could observe the impact of each single control variable, in order to determine the control parameter range. This pre-evaluation provides a full factorial design with the variable factors and factor levels presented in Table 1, which is then used to study the influence of the input parameters on the finished workpiece, for which the response parameters are surface roughness $[\mu \mathrm{m}]$, the $\operatorname{MRR}[\mathrm{mm} 3 / \mathrm{min}]$ and the width and depth dimensions [ $\mu \mathrm{m}]$.

Table 1. Variable factors and factor levels.

\begin{tabular}{|c|c|c|c|}
\hline Variable Factors & \multicolumn{3}{|c|}{ Factor Levels } \\
\hline \multirow{3}{*}{ Scanning Speed (SS) [mm/s] } & 200 & 225 & 250 \\
\cline { 2 - 4 } & 275 & 300 & 325 \\
\cline { 2 - 4 } & 350 & 375 & 400 \\
\hline Pulse Intensity (PI) [\%] & 35 & 40 & 45 \\
\hline Pulse Frequency (PF)[kHz] & 35 & 40 & - \\
\hline
\end{tabular}




\section{Experimental results and discussion}

Following the design of the experiments summarized in Table 1, 54 micro channels were machined with the laser machining process. Surface roughness was measured in five different sections of each micro-channel to get the mean value of the entire channel. Then, each channel was cut into three parts to get the cross-section profiles, from which the measurements of depth and width were taken by processed digital images. Once again, five different measurements, proportionally distributed along the depth and the width, were measured. The removed area was also measured for each channel profile, in order to calculate the material removal rate. The mean values of the experimental results obtained from the machined features for all the combinations of the variable factors are shown in Table 2 . The micro-channels presented variations in dimensions and shape. These variations are clearly represented in Figure 2, which presents the images of six micro-channels. Analysis of Variance was performed for each response factor to study the influence of the process parameters.

Table 2. DoE with the experimental results.

\begin{tabular}{|c|c|c|c|c|c|c|c|c|c|c|c|c|c|c|c|}
\hline \# & $\begin{array}{l}\text { PI } \\
(\%)\end{array}$ & $\begin{array}{c}\text { PF } \\
(\mathrm{kHz})\end{array}$ & $\begin{array}{c}\text { SS } \\
(\mathrm{mm} / \mathrm{s})\end{array}$ & $\begin{array}{l}\text { depth } \\
(\mu \mathrm{m})\end{array}$ & $\begin{array}{l}\text { width } \\
(\mu \mathrm{m})\end{array}$ & $\begin{array}{c}\mathrm{Ra} \\
(\mu \mathrm{m})\end{array}$ & $\begin{array}{c}\text { MRR } \\
\left(\mathrm{mm}^{3} / \mathrm{min}\right)\end{array}$ & \# & $\begin{array}{l}\text { PI } \\
(\%)\end{array}$ & $\begin{array}{c}\text { PF } \\
(\mathrm{kHz})\end{array}$ & $\begin{array}{c}\text { SS } \\
(\mathrm{mm} / \mathrm{s})\end{array}$ & $\begin{array}{c}\text { depth } \\
(\mu \mathrm{m})\end{array}$ & $\begin{array}{l}\text { width } \\
(\mu \mathrm{m})\end{array}$ & $\begin{array}{c}\mathrm{Ra} \\
(\mu \mathrm{m})\end{array}$ & $\begin{array}{c}\text { MRR } \\
\left(\mathrm{mm}^{3} / \mathrm{min}\right)\end{array}$ \\
\hline 1 & 35 & 35 & 200 & 18.3 & 189 & 0.505 & 0.034 & 28 & 35 & 40 & 200 & 13.9 & 192.9 & 0.560 & 0.025 \\
\hline 2 & 35 & 35 & 225 & 17.4 & 190 & 0.477 & 0.036 & 29 & 35 & 40 & 225 & 12.6 & 175.5 & 0.479 & 0.017 \\
\hline 3 & 35 & 35 & 250 & 14.9 & 191 & 0.533 & 0.034 & 30 & 35 & 40 & 250 & 11.6 & 188.1 & 0.531 & 0.026 \\
\hline 4 & 35 & 35 & 275 & 15.7 & 195.8 & 0.455 & 0.041 & 31 & 35 & 40 & 275 & 15.7 & 191.6 & 0.465 & 0.034 \\
\hline 5 & 35 & 35 & 300 & 12.9 & 197.7 & 0.456 & 0.033 & 32 & 35 & 40 & 300 & 8.1 & 193.2 & 0.506 & 0.026 \\
\hline 6 & 35 & 35 & 325 & 11.6 & 193.2 & 0.463 & 0.027 & 33 & 35 & 40 & 325 & 8.1 & 189.8 & 0.520 & 0.020 \\
\hline 7 & 35 & 35 & 350 & 8.1 & 191.7 & 0.470 & 0.019 & 34 & 35 & 40 & 350 & 11.6 & 189.2 & 0.471 & 0.034 \\
\hline 8 & 35 & 35 & 375 & 10.9 & 192.5 & 0.504 & 0.021 & 35 & 35 & 40 & 375 & 10.8 & 189.9 & 0.525 & 0.027 \\
\hline 9 & 35 & 35 & 400 & 10.2 & 192.8 & 0.457 & 0.027 & 36 & 35 & 40 & 400 & 11.7 & 190.1 & 0.463 & 0.027 \\
\hline 10 & 40 & 35 & 200 & 29.9 & 183.9 & 0.549 & 0.055 & 37 & 40 & 40 & 200 & 31.2 & 186.1 & 0.531 & 0.065 \\
\hline 11 & 40 & 35 & 225 & 30.0 & 184.9 & 0.481 & 0.059 & 38 & 40 & 40 & 225 & 26.2 & 186.6 & 0.571 & 61 \\
\hline 12 & 40 & 35 & 250 & 25.4 & 184.4 & 0.513 & 0.061 & 39 & 40 & 40 & 250 & 23.7 & 187.3 & 0.462 & 0.056 \\
\hline 13 & 40 & 35 & 275 & 21.9 & 187.2 & 0.664 & 0.048 & 40 & 40 & 40 & 275 & 17.1 & 190.4 & 0.510 & 0.026 \\
\hline 14 & 40 & 35 & 300 & 16.8 & 189.9 & 0.478 & 0.036 & 41 & 40 & 40 & 300 & 17.7 & 195.7 & 0.459 & 0.041 \\
\hline 15 & 40 & 35 & 325 & 14.4 & 188.4 & 0.473 & 0.032 & 42 & 40 & 40 & 325 & 19.2 & 192.3 & 0.461 & 0.061 \\
\hline 16 & 40 & 35 & 350 & 18.5 & 188.5 & 0.485 & +1 & 43 & 40 & 40 & 350 & 17.3 & 190.3 & 0.435 & .039 \\
\hline 17 & 40 & 35 & 375 & 18.2 & 190.5 & 0.457 & 0.048 & 44 & 40 & 40 & 375 & 16.5 & 190.5 & 0.490 & 0.044 \\
\hline 18 & 40 & 35 & 400 & 18.4 & 190.0 & 0.382 & 0.043 & 45 & 40 & 40 & 400 & 14.2 & 192.3 & 0.423 & 0.040 \\
\hline 19 & 45 & 35 & 200 & 39.6 & 184.4 & 0.519 & 0.065 & 46 & 45 & 40 & 200 & 38.6 & 184.4 & 0.519 & 0.074 \\
\hline 20 & 45 & 35 & 225 & 35.8 & 184.1 & 0.513 & 0.073 & 47 & 45 & 40 & 225 & 35.0 & 184.2 & 0.531 & 0.067 \\
\hline 21 & 45 & 35 & 250 & 33.7 & 181.0 & 0.493 & 0.072 & 48 & 45 & 40 & 250 & 29.5 & 180.7 & 0.526 & 0.071 \\
\hline 22 & 45 & 35 & 275 & 22.1 & 184.3 & 0.443 & 0.063 & 49 & 45 & 40 & 275 & 26.8 & 185.3 & 0.523 & 0.056 \\
\hline 23 & 45 & 35 & 300 & 25.4 & 186.2 & 0.451 & 0.057 & 50 & 45 & 40 & 300 & 25.1 & 187 & 0.514 & 0.067 \\
\hline 24 & 45 & 35 & 325 & 26.5 & 189.2 & 0.451 & 0.061 & 51 & 45 & 40 & 325 & 22.8 & 186.8 & 0.446 & 0.062 \\
\hline 25 & 45 & 35 & 350 & 20.8 & 191.1 & 0.447 & 0.047 & 52 & 45 & 40 & 350 & 19.3 & 187.3 & 0.509 & 0.039 \\
\hline 26 & 45 & 35 & 375 & 19.8 & 189.9 & 0.397 & 0.054 & 53 & 45 & 40 & 375 & 17.5 & 187.6 & 0.408 & 0.040 \\
\hline
\end{tabular}




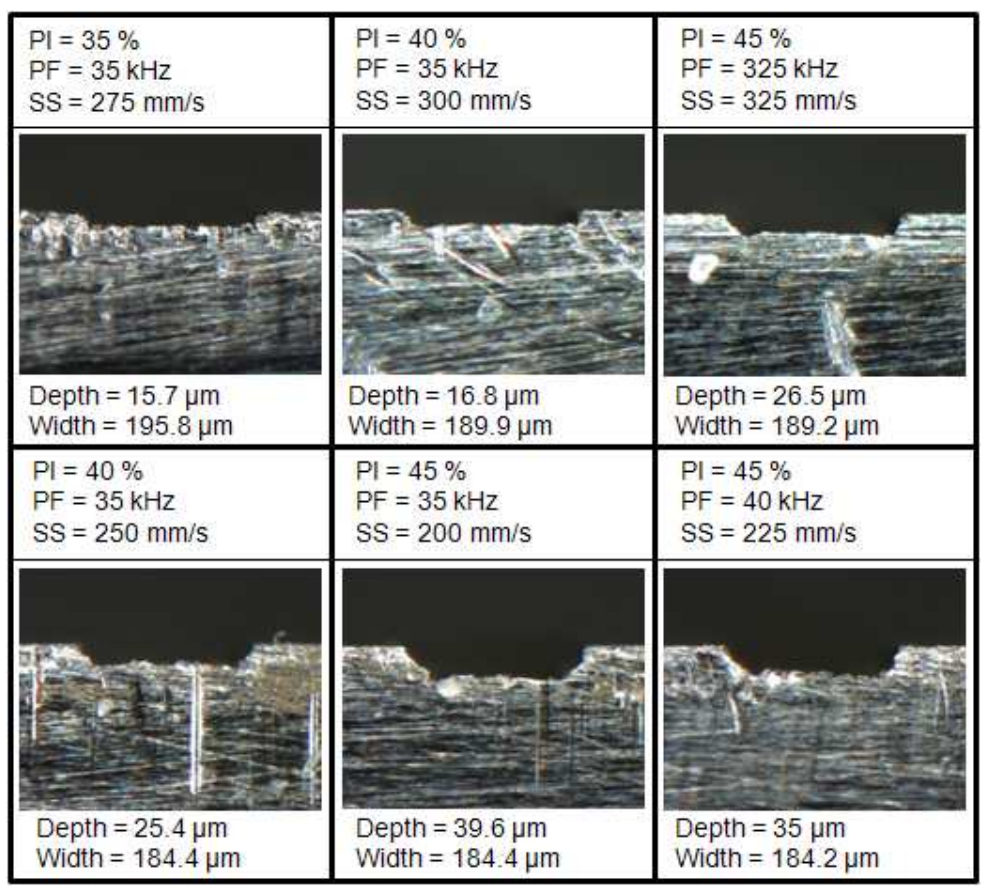

Figure 2. Images of micro-channels

\subsection{Micro-channel depth}

As can be seen in Table 2, which shows the results obtained for the micro-channel depths, the target depth of $50 \mu \mathrm{m}$ was never reached. This is clear in Figure 3, where the influence of the scanning speed and the pulse intensity on the depth dimension is summarized. It shows that almost all of the machined depths are within the 10 to $25 \mu \mathrm{m}$ range and only few experiments achieved depth values above this range.

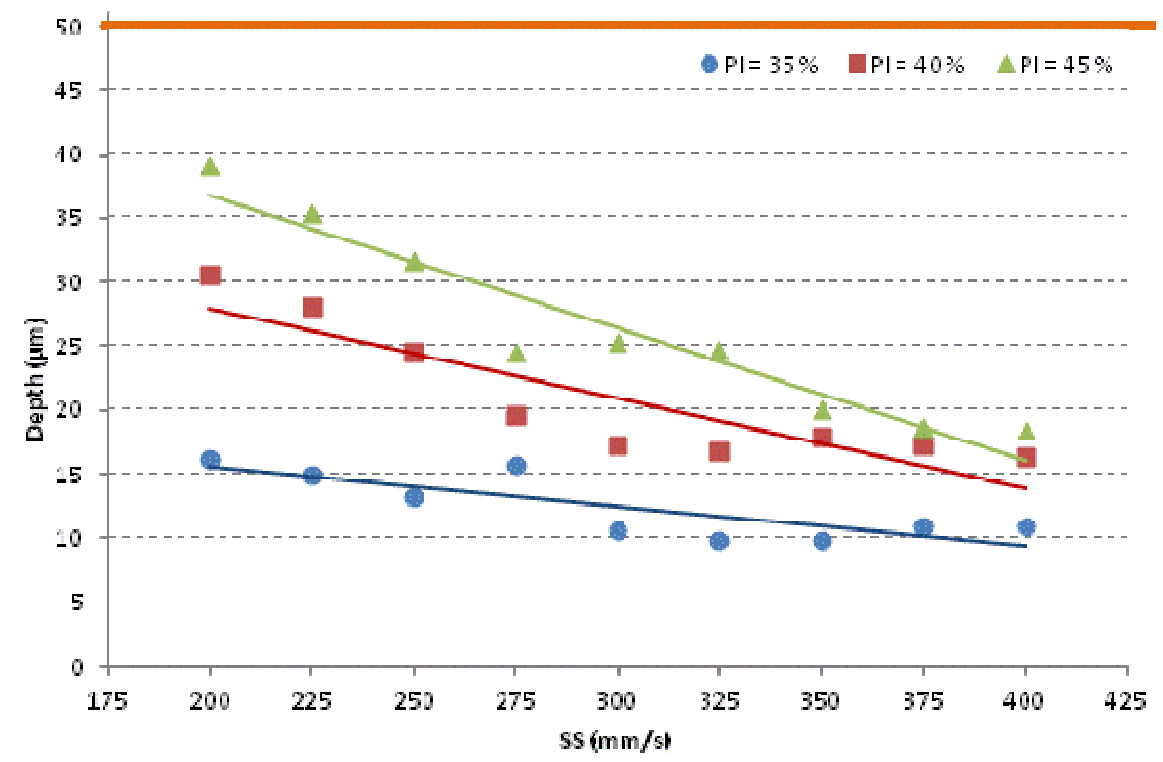

Figure 3. Influence of scanning speed and pulse intensity on depth dimension. 
The trend lines presented in Figure 3 clearly show that higher scanning speeds result in smaller depths and higher pulse intensities result in deeper micro-channels. Thus, the greatest depth was reached with the lowest scanning speed $(200 \mathrm{~mm} / \mathrm{s})$ and the highest pulse intensity $(45 \%)$. In a laser milling process (with 3D movements), slower displacements of the laser beam means that the surface is machined with high energy for a longer time, which allows a larger amount of energy to be absorbed by the material resulting in channels of greater depths. This demonstrates that higher pulse intensity values would be necessary to obtain depth values closer to the target.

Table 3 summarizes the results of the ANOVA revealing that the most significant factors for the average depth of micro-channels are scanning speed and pulse intensity, as previously pointed out $(p<0.005)$. The F-values indicate that pulse intensity is the most significant factor, which is made clearer by the contribution values.

Table 3. ANOVA analysis for depth.

\begin{tabular}{ccccccc}
\hline Factor & $\begin{array}{c}\text { Degrees of } \\
\text { freedom } \\
\text { (DOF) }\end{array}$ & $\begin{array}{c}\text { Sum of } \\
\text { squares } \\
\text { (SS) }\end{array}$ & $\begin{array}{c}\text { Mean } \\
\text { squares } \\
\text { (MS) }\end{array}$ & F-value & P-value & $\begin{array}{c}\text { Contribution } \\
\text { (\%) }\end{array}$ \\
\hline $\boldsymbol{P I}$ & 2 & 1775.26 & 887.63 & 94.72 & 0.000 & 83.9 \\
$\boldsymbol{P F}$ & 1 & 25.43 & 25.43 & 2.71 & 0.107 & 2.4 \\
SS & 8 & 1163.59 & 145.45 & 15.52 & 0.000 & 13.7 \\
Residual & 42 & 393.61 & 9.37 & - & - & - \\
\hline
\end{tabular}

Compared with other authors, the experimental results shows that higher pulse intensity and lower scanning speeds tend to give deeper channels, which is in line with the idea that the number of pulses per $\mathrm{mm}$ increases, as the laser beam moves more slowly across the workpiece, thus removing more material. Furthermore, when the intensity is higher, the pulse energy increases, which in turn results in greater depth values (Bordatchev, 2003 and Youseff, 2003).

\subsection{Micro-channel width}

Table 2 presents width dimensions that range from 175.5 to $197.7 \mu \mathrm{m}$. Once again, no experiment achieves the target value $(200 \mu \mathrm{m})$. Figure 4 shows how the scanning speed and the pulse intensity affect the average width. In this case, in contrast to the results on depth, the experimental values are closer to the target width when the scanning speed is high and the pulse intensity is low. These converse effects on width and depth are due to the fact that straight walls are really difficult to achieve. Thus, as the channel becomes deeper, the width becomes narrower, producing a smaller mean width value. 
Table 4 summarizes the results of the ANOVA analysis on the average width. It can be seen that the pulse frequency has no statistically significant effect on width dimension. The parameters that do have a significant effect on the width are pulse intensity and scanning speed, with pulse intensity being the most significant, as is clearly indicated by the results of the F-value, with a contribution of $69.5 \%$

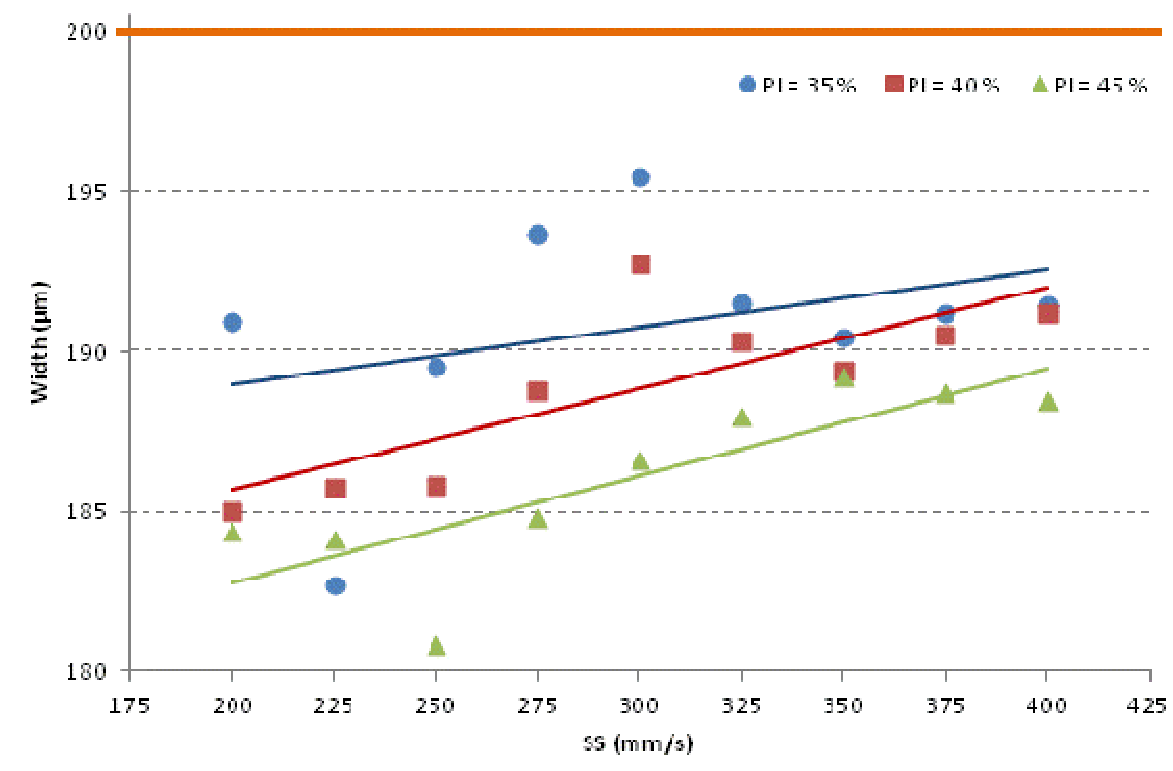

Figure 4. Influence of scanning speed and pulse intensity on width dimension.

Table 4. ANOVA analysis for width.

\begin{tabular}{ccccccc}
\hline Factor & $\begin{array}{c}\text { Degrees of } \\
\text { freedom } \\
\text { (DF) }\end{array}$ & $\begin{array}{c}\text { Sum of } \\
\text { squares } \\
\text { (SS) }\end{array}$ & $\begin{array}{c}\text { Mean } \\
\text { squares } \\
\text { (MS) }\end{array}$ & $\boldsymbol{F}$ & $\boldsymbol{P}$ & $\begin{array}{c}\text { Contribution } \\
\text { (\%) }\end{array}$ \\
\hline $\boldsymbol{P I}$ & 2 & 195.88 & 97.94 & 12.15 & 0.000 & 69.5 \\
PF & 1 & 5.01 & 5.01 & 0.62 & 0.435 & 3.5 \\
SS & 8 & 304.22 & 38.03 & 4.72 & 0.000 & 27 \\
Residual & 42 & 338.43 & 8.06 & - & - & - \\
\hline
\end{tabular}

Although other studies using single laser shots (Bordatchev, 2003 and Youseff, 2003) have concluded that crater depth and diameter increase with pulse energy, in our case, the width decreases. This effect can be explained because in the laser milling process several passes are necessary along all axes to obtain the final shape. Thus, because of the difficulty in achieving straight walls due the Gaussian shape of the laser beam, as the channel gets deeper, the width narrows. Therefore, the mean width of the channel decreases. 


\subsection{Micro-channel surface roughness}

The influence of the variable factors on surface roughness was also evaluated. Figure 5 shows the effect of the scanning speed and the pulse intensity on surface roughness. The trend lines indicate that surface roughness decreases at high scanning speeds. The influence of pulse intensity is less clear, although it does seem to indicate that higher intensity results in lower surface roughness. Slow scanning speeds do not improve surface roughness and fast movements hardly affect it. Furthermore, the experimental results show no large differences, with a range between 0.4 and $0.55 \mu \mathrm{m}$. The best surface roughness values were obtained with a combination of the highest pulse intensity and highest scanning speed.

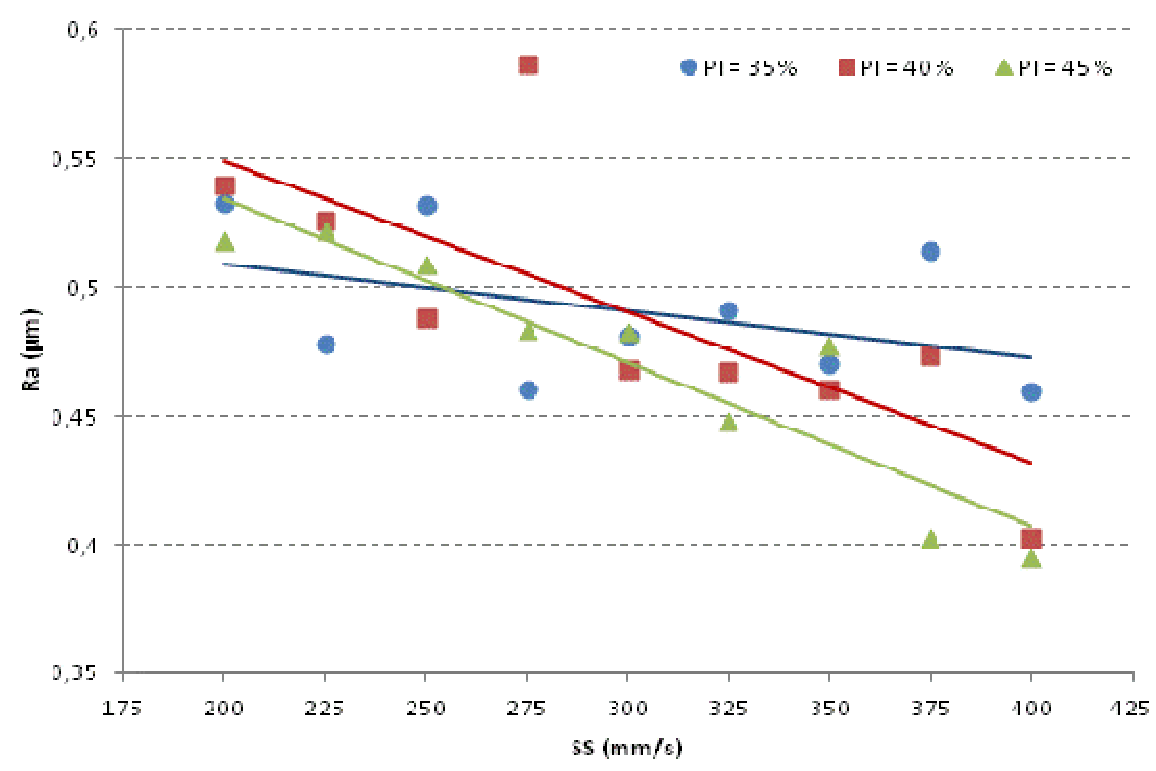

Figure 5. Influence of scanning speed and pulse intensity on surface roughness.

Table 5 summarizes the results of the ANOVA, which reveal that scanning speed is the most significant factor in surface roughness, while neither pulse intensity nor pulse frequency has a statistically significant effect on surface roughness. However, the contribution of the scanning speed is relatively small at $46.1 \%$.

Table 5. ANOVA analysis for surface roughness.

\begin{tabular}{ccccccc}
\hline Factor & $\begin{array}{c}\text { Degrees of } \\
\text { freedom } \\
\text { (DF) }\end{array}$ & $\begin{array}{c}\text { Sum of } \\
\text { squares } \\
\text { (SS) }\end{array}$ & $\begin{array}{c}\text { Mean } \\
\text { squares } \\
\text { (MS) }\end{array}$ & $\boldsymbol{F}$ & $\boldsymbol{P}$ & $\begin{array}{c}\text { Contribution } \\
\text { (\%) }\end{array}$ \\
\hline $\boldsymbol{P I}$ & 2 & 0.004 & 0.002 & 1.92 & 0.160 & 13.4 \\
PF & 1 & 0.005 & 0.005 & 5.79 & 0.021 & 40.5 \\
SS & 8 & 0.051 & 0.006 & 6.58 & 0.000 & 46.1 \\
Residual & 42 & 0.039 & 0.001 & - & - & - \\
\hline
\end{tabular}


The experimental results shows that high pulse intensities and slower scanning speeds mean that more energy is applied to the workpiece, increasing the damage caused to the surface. Therefore, lower pulse intensities and higher scanning speeds will improve the final quality of the machined parts, because surface roughness will be reduced (Bordatchev, 2003).

\subsection{Micro-channel material removal rate}

The effect of the process parameters on the MRR was also studied. Figure 6 presents the effects of pulse intensity and scanning speed on the MRR. The trend lines clearly indicate that MRR increases with lower scanning speeds and higher pulse intensities. Although higher scanning speeds result in faster processes, the area of material removal is smaller, thus the MRR decreases. On the other hand, higher pulse intensity results in deeper channels and, in consequence, higher MRRs.

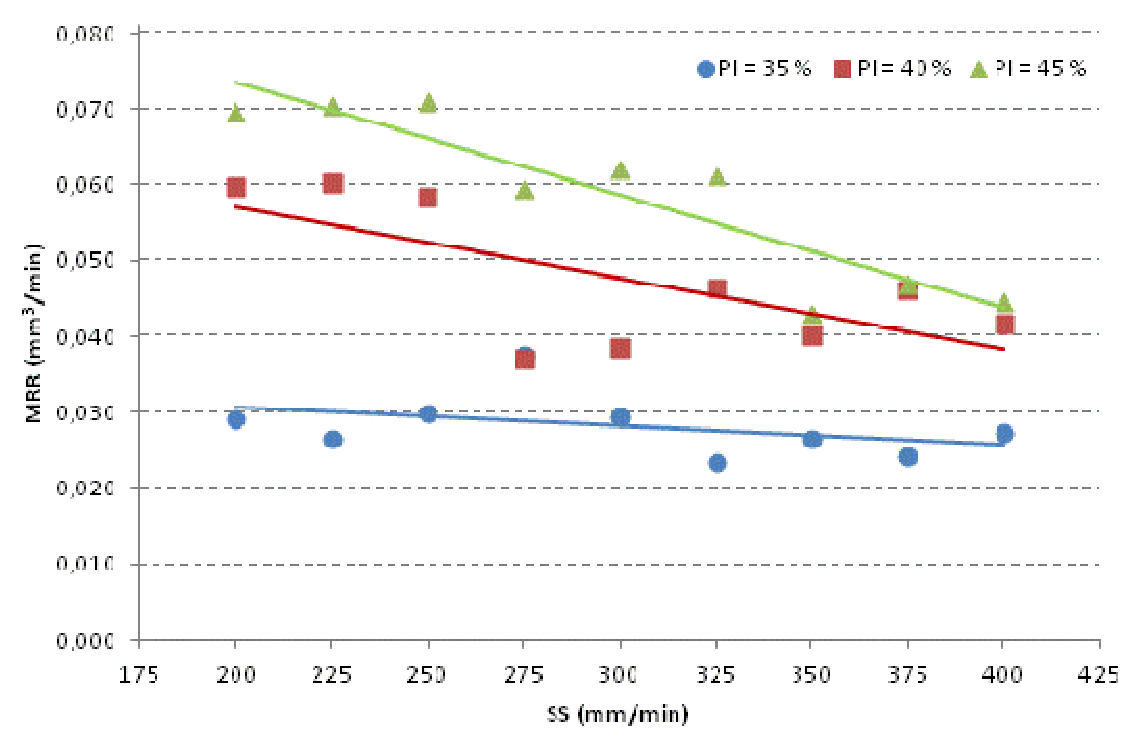

Figure 6. Influence of scanning speed and pulse intensity on MRR.

The ANOVA results for MRR are shown in Table 6. It can be seen that pulse frequency has no statistically significant effect on MRR. Pulse intensity is found to have the most significant effect on MMR with a contribution of $93.1 \%$, while scanning speed has a somewhat lesser impact, with a contribution of $5.9 \%$.

Table 6. ANOVA analysis for MRR.

\begin{tabular}{cccccccc}
\hline Factor & $\begin{array}{c}\text { Degrees of } \\
\text { freedom } \\
\text { (DF) }\end{array}$ & $\begin{array}{c}\text { Sum of } \\
\text { squares } \\
\text { (SS) }\end{array}$ & $\begin{array}{c}\text { Mean } \\
\text { squares } \\
\text { (MS) }\end{array}$ & $\boldsymbol{F}$ & $\boldsymbol{P}$ & $\begin{array}{c}\text { Contribution } \\
\text { (\%) }\end{array}$ \\
\hline $\boldsymbol{P I}$ & 2 & 0.0086 & 0.0043 & 57.92 & 0.000 & 93.1 \\
$\boldsymbol{P F}$ & 1 & $4.82 \cdot 10^{-5}$ & $4.82 \cdot 10^{-5}$ & 0.65 & 0.424 & 1 \\
SS & 8 & 0.0021 & 0.0002 & 3.65 & 0.003 & 5.9 \\
Residual & 42 & 0.0031 & $7.4 \cdot 10^{-5}$ & - & - & - \\
\hline
\end{tabular}


Since MRR is directly proportional to the width and depth of the channel, the MRR plot has a shape that is similar to the depth plot, due to the fact that the influence of the depth is greater than that of the width.

\section{Modeling}

Following the experimental tests and the study and ANOVA analysis of the relationship between the parameters, various machine learning techniques were then selected and tested for the depth and width dimension, surface roughness and MRR, in order to determine their correlation with the process parameters. The objective was to obtain the most appropriate process parameters for producing minimal surface roughness with the highest material removal rate. This selection included the k-Nearest Neighbors (kNN) technique with $\mathrm{k}$ set to $1, \ldots, 5$, linear regression, decision trees, and multilayer perceptrons. Hence, these methods were considered, which have clear decision rules and the capability to perform both linear and nonlinear transformations on the input data. A 10 fold cross-validation was applied, which takes account of the capability of the models to predict output parameter values from new input data. A naïve approach was adopted as a baseline, to ensure that the new models are extracting useful information from the dataset. The correlation coefficient (R2) and Mean Absolute Error (MAE) for individual input parameters modeled with the naïve approach were analyzed. The results are shown in Table 7, in which it may be seen that the correlation coefficient for the four models with the naïve approach is very low.

$\begin{array}{ccc}\text { Table } 7 . \text { Naive prediction for individual parameters. } \\ \text { Parameter } & \text { Correlation coefficient }\left(\boldsymbol{R}^{2}\right) & \text { Mean absolute error (MAE) } \\ \text { Depth } & 0.178 & 6.49 \\ \text { Width } & 0.133 & 3.078 \\ \text { Surface Roughness }\left(\mathbf{R}_{\alpha}\right) & 0.239 & 0.0397 \\ \text { MRR } & 0.178 & 0.0141\end{array}$

Starting with the low accuracy provided by a naïve approach, different machine learning models were built for each output parameter. First, the accuracy, in terms of R2 and MAE, of the depth modelling are shown in Table 8. The best results are observed for decision trees (in bold in Table 8), which yield the highest correlation coefficient and lowest MAE out of all analyzed models. It can be observed that $1 \mathrm{NN}$ is the best technique out of all the analyzed kNN simulations. This suggests limited noisiness of depth, i.e. the most similar input features provide the best estimation of the output parameter. It is interesting to investigate the definition of the tree shown in Fig. 7. As is clear from the ANOVA analysis, pulse intensity is the main parameter $(83.9 \%)$ for this process, therefore the first node refers to this parameter; scanning speed is the second parameter from the ANOVA analysis (13.7\%) and forms the 
second level nodes of the tree; leaving the last level for the lower influence: the pulse frequency. It is also interesting how the tree generates the final leaves at different scanning speeds depending on the pulse intensity, a conclusion that was expected considering the relation between both parameters shown in Figure 3: this capability of decision trees, which produce clear graphical conclusions on the influence of each parameter, makes them the most accurate technique. Linear regression models do not also achieve the required accuracy; this result fits well with the conclusion presented in Section 3 that channel depth depends mainly on scanning speed and pulse intensity and that this dependency is not linear.

\begin{tabular}{ccccccccc} 
& \multicolumn{8}{c}{ Table 8. Accuracy of different models of laser milling depth } \\
& $\mathbf{1 N N}$ & $\mathbf{2 N N}$ & $\mathbf{3 N N}$ & $\mathbf{4 N N}$ & $\mathbf{5 N N}$ & $\begin{array}{c}\text { Linear } \\
\text { regression }\end{array}$ & $\begin{array}{c}\text { Decision } \\
\text { tree }\end{array}$ & $\begin{array}{c}\text { Multilayer } \\
\text { perceptron }\end{array}$ \\
$\mathbf{R}^{2}$ & 0.870 & 0.870 & 0.819 & 0.799 & 0.807 & 0.82 & $\mathbf{0 . 9 5 5}$ & 0.940 \\
MAE & 2.04 & 2.04 & 2.60 & 2.81 & 2.80 & 2.90 & $\mathbf{1 . 3 1}$ & 1.44
\end{tabular}

Figure 7. Decision tree for the estimation of depth

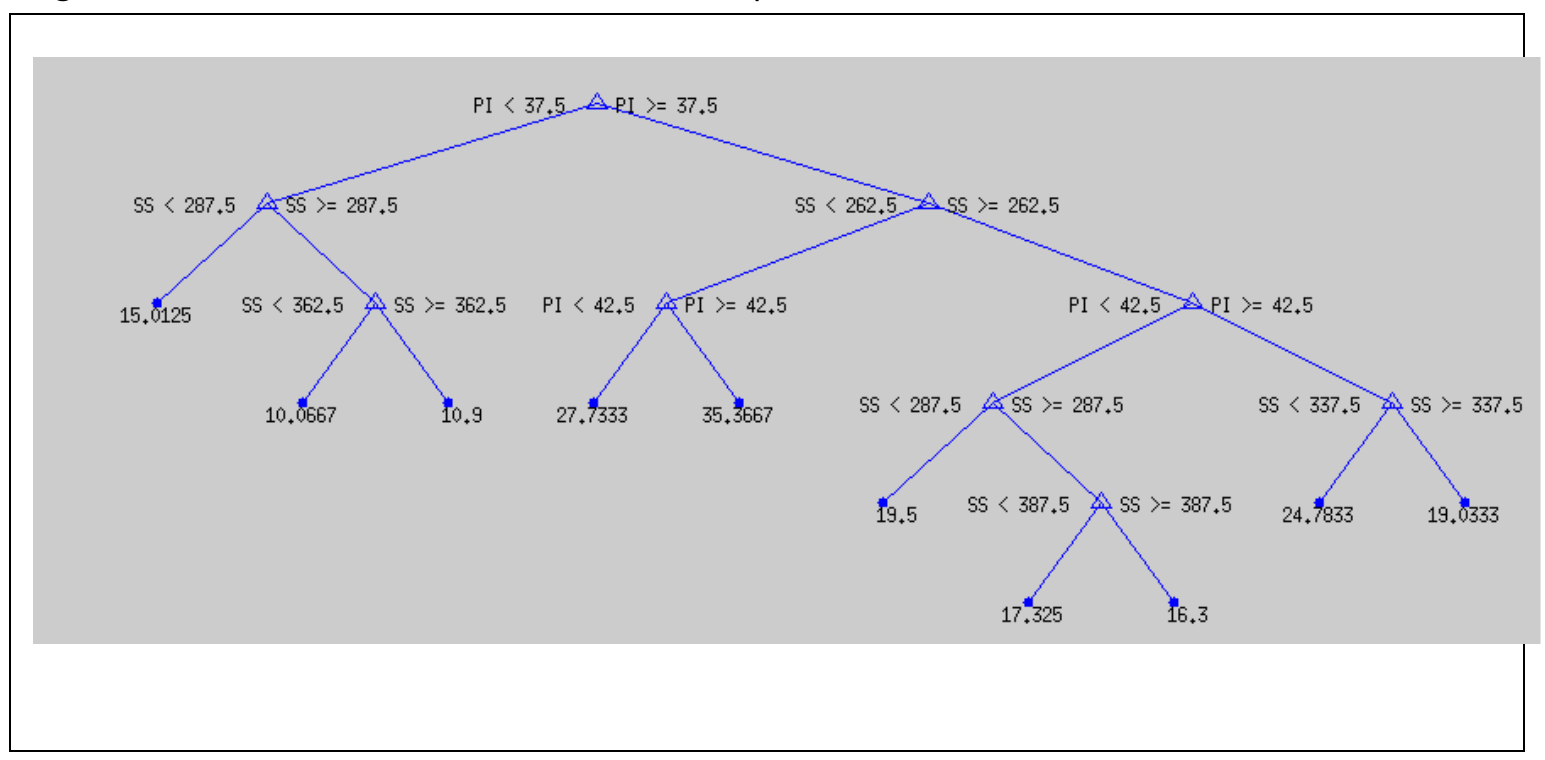

Next, the results for width were analyzed. These are shown in Table 9. The best method turns out to be a multilayer perceptron (in bold in Table 9), which suggests that a nonlinear method is needed this time. It can be observed than the $3 \mathrm{NN}$ method provides the best correlation of all the kNN simulations that were analyzed. However, the lowest MAE rate for kNN methods is observed for $k=1$. As already observed for width modelling, linear regression models do not achieve the expected accuracy; this result fits well with the conclusion presented in Section 3, that channel width depends mainly on scanning speed and pulse intensity and that this dependency is clearly not linear. 


\begin{tabular}{|c|c|c|c|c|c|c|c|c|}
\hline & $1 N N$ & $2 N N$ & $3 N N$ & $4 N N$ & $5 N N$ & $\begin{array}{c}\text { Linear } \\
\text { regression }\end{array}$ & $\begin{array}{c}\text { Decision } \\
\text { tree }\end{array}$ & $\begin{array}{l}\text { Multilayer } \\
\text { perceptron }\end{array}$ \\
\hline$R^{2}$ & 0.325 & 0.325 & 0.393 & 0.364 & 0.348 & 0.367 & 0.371 & 0.606 \\
\hline MAE & 1.82 & 1.82 & 1.95 & 2.07 & 2.14 & 2.09 & 2.18 & 1.80 \\
\hline
\end{tabular}

In the case of surface roughness modeling, an interesting phenomenon is observed (Tables 10 and 11). The correlation coefficient values appear to grow with higher values of k. Hence, extended analysis for the values of k exceeding 5 was conducted, as shown in Table 11.

\begin{tabular}{|c|c|c|c|c|c|c|c|c|}
\hline & $1 N N$ & $2 N N$ & $3 N N$ & $4 N N$ & $5 N N$ & $\begin{array}{c}\text { Linear } \\
\text { regression }\end{array}$ & $\begin{array}{c}\text { Decision } \\
\text { tree }\end{array}$ & $\begin{array}{l}\text { Multilayer } \\
\text { perceptron }\end{array}$ \\
\hline$R^{2}$ & 0.0903 & 0.0903 & 0.2053 & 0.2361 & 0.3053 & 0.323 & 0.5952 & 0.4809 \\
\hline AAE & 0.043 & 0.043 & 0.035 & 0.034 & 0.033 & 0.030 & 0.023 & 0.029 \\
\hline
\end{tabular}

As expected, the value of correlation coefficient deteriorates for $k>5$. Obviously, it will converge to naïve results that are guessed. The main conclusion here is that the impact of process settings on surface roughness is quite sophisticated and possibly noisy, as averaging roughness from the most similar experiments to $\mathrm{K}=5$ yields the best roughness prediction out of all kNN experiments. At the same time, the best overall correlation coefficient value and MAE rate is attained for decision trees (in bold in Table 10) and is largely superior to kNN. As already observed for width and depth modelling, linear regression models do not achieve the expected accuracy. This result fits well with the conclusion in Section 3, which states that channel roughness depends mainly on scanning speed with a very noisy dependency, which in no case is ever linear.

\begin{tabular}{|c|c|c|c|c|c|c|c|c|c|c|}
\hline & $6 N N$ & $7 N N$ & $8 N N$ & 9NN & $10 N N$ & $11 N N$ & $12 N N$ & $13 N N$ & $14 N N$ & $15 N N$ \\
\hline $\mathbf{R}^{2}$ & 0.242 & 0.231 & 0.263 & 0.264 & 0.240 & 0.152 & 0.116 & 0.100 & 0.096 & 0.090 \\
\hline MAE & 0.034 & 0.033 & 0.032 & 0.033 & 0.034 & 0.036 & 0.037 & 0.037 & 0.037 & 0.03 \\
\hline
\end{tabular}

In the case of MRR modelling, Table 12, multilayer perceptrons and decision trees yield the best and virtually identical results (in bold in Table 12). In this case, 1NN proves to be the best method out of kNN methods for $\mathrm{k}$ ranging from 1 to 5 . This is in line with previous findings for depth i.e. the closest input settings produce the most similar output parameter value: this 
time, the MRR rates. Linear regression models do not achieve the required accuracy; this result fits well with the conclusion presented in Section 3, that MRR depends mainly on pulse intensity (more than 93\% in the ANOVA test) and that this dependency is not linear.

\begin{tabular}{|c|c|c|c|c|c|c|c|c|}
\hline & $1 N N$ & $2 N N$ & $3 N N$ & $4 N N$ & $5 N N$ & $\begin{array}{l}\text { Linear } \\
\text { regression }\end{array}$ & $\begin{array}{c}\text { Decision } \\
\text { tree }\end{array}$ & $\begin{array}{l}\text { Multilayer } \\
\text { perceptron }\end{array}$ \\
\hline$R^{2}$ & 0.769 & 0.769 & 0.680 & 0.650 & 0.657 & 0.702 & 0.825 & 0.828 \\
\hline MAE & 0.006 & 0.006 & 0.007 & 0.007 & 0.008 & 0.007 & 0.005 & 0.005 \\
\hline
\end{tabular}

To sum up, prediction accuracy evaluation, depth and MRR can be modeled with high accuracy. Lower, but still significant accuracy is observed for surface roughness and width modelling. In the case of surface roughness, a higher value of $k$, meaning the averaging of roughness based on many similar experiments gives better results than using the roughness from the most similar experiment in terms of input settings. This fact suggests noisiness of the data, dependencies between inputs and outputs that are difficult to capture and even the need to collect other parameter values that contribute to the problem. In accordance with the bibliography (Bernardos 2003), it can be concluded that surface roughness depends on too many variables to assure a complete determination of the milling process and therefore models are expected to be, in any case, less accurate than the other performance parameters under study. In no case did the linear regressions show high accuracy, a result that was expected, considering the non-linear dependencies between input and output parameters in all cases.

\section{Conclusions}

Micro-laser milling is a machining process suitable for fabricating micro-moulds. However, it requires the most appropriate process parameters settings. In this study, the surface quality, dimensional features and productivity of micro-channels have been studied in a micro laser milling process. Although the results obtained for the micro-channels present variations, they do suggest that laser machining is capable of producing micro-geometries. Several specific conclusions should be pointed out:

1. Low scanning speeds and high pulse intensities increase the depth and decrease the width of the micro-channels.

2. The surface quality of the channels improves with a rise in scanning speed, which in turn decreases surface roughness.

3. Laser micromachining productivity increases with high pulse intensities and low scanning speeds.

4. ANOVA results show that pulse frequency is not statistically significant for the responses under study. 
5. Machine learning techniques are suitable techniques with which to model laser-milling manufacture of micro shapes. Higher accuracy is observed for surface roughness and width modelling, than for depth and material removal rate modelling. Neural networks were better at modelling width dimensions and decision trees were better at modelling surface roughness; both techniques were similar for depth and material removal rate. In all cases these two techniques showed better accuracy than the other two models: k-nearest neighbors and linear regression. The use of decision trees is therefore feasible, if the dimensional accuracy of the workpiece is the main industrial requirement, while neural networks are better in the other cases.

6. The Nearest Neighbor models with higher $k$ values show greater accuracy for roughness prediction, allowing us conclude that the noisiness of this output is higher or that it depends on more parameters than the other variables, as suggested in the previous literature.

Future work will consider other Al techniques, such as ensembles of classifiers or regressors. These ensembles are built by combining different basic classifiers that could improve final model accuracy. This experimental methodology, in which the best process parameter combination is selected, will also be applied to other types of materials, such as transparent polymers typically used for disposable lab-on-chip devices and ceramics used for various industrial applications in aeronautics, automobile manufacturing, electronics, medicine and semiconductors.

\section{Acknowledgments}

This work was partially carried out with the grant supports from the European Commission project IREBID (FP7-PEOPLE-2009-IRSES-247476) and the Spanish Science and Innovation Minister project TECNIPLAD (DPI2009- 09852).

\section{References}

Bartolo P, Vasco J, Silva B, Galo C. Laser micromachining for mould manufacturing: I. The influence of operating parameters. Assem Autom 2006;26(3):227-234.

Benardos P.G., Vosniakos G.C., "Predicting surface roughness in machining: a review", Int J Mach Tools Manuf 43(8), 2003, 833-844.

Bordatchev EV, Nikumb SK. An experimental study and statistical analysis of the effect of laser pulse energy on the geometric quality during laser precision machining. Mach Sci Technol 2003;7(1):83-104.

Brousseau E., Eldukhri E., "Recent advances on key technologies for innovative manufacturing", Journal of Intelligent Manufacturing, 22(5), 2011, 675-691. 
Bustillo A., Díez-Pastor J.F., Quintana G. and García-Osorio C., "Avoiding neural network fine tuning by using ensemble learning: application to ball-end milling operations", The International Journal of Advanced Manufacturing Technology, 57(5), 2011, 521-532.

Bustillo A., Ukar E., Rodriguez J. J., Lamikiz A. "Modelling of process parameters in laser polishing of steel components using ensembles of regression trees", International Journal of Computer Integrated Manufacturing, 24(8), 2011, 735-747.

Bustillo A., Correa M., "Using artificial intelligence to predict surface roughness in deep drilling of Steel Components", Journal of Intelligent Manufacturing, 23(5), 2012, 1893-1902.

Campanelli SL, Ludovico AD, Bonserio C, Cavalluzzi P, Cinquepalmi M. Experimental analysis of the laser milling process parameters. J Mater Process Technol 2007 8/1;191(1-3):220-223.

Chandrasekaran M, Muralidhar M, Krishna C, Dixit U "Application of soft computing techniques in machining performance prediction and optimization: a literature review". Int J Adv Manuf Technol 46(5), 2010, 445-464.

Cicală E, Soveja A, Sallamand P, Grevey D, Jouvard JM. The application of the random balance method in laser machining of metals. J Mater Process Technol 2008 1/21;196(1-3):393-401.

Ciurana J, Arias G, Ozel T. Neural network modeling and particle swarm optimization (PSO) of process parameters in pulsed laser micromachining of hardened AISI H13 steel. Mater Manuf Process 2009;24(3):358-368.

Correa, M., Bielza, C., \& Pamies-Teixeira, J. "Comparison of Bayesian networks and artificial neural networks for quality detection in a machining process", Expert Systems with Applications, 36, 2009, 7270-7279.

Desai C. K., Shaikh A., "Prediction of depth of cut for single-pass laser micro-milling process using semi-analytical, ANN and GP approaches" International Journal Of Advanced Manufacturing Technology, 60(9-12), 2012, 865-882.

Dhara SK, Kuar AS, Mitra S. An artificial neural network approach on parametric optimization of laser micro-machining of die-steel. Int J Adv Manuf Technol 2008;39(1-2):39-46.

Díez-Pastor J. F., Bustillo A., Quintana G. and García-Osorio C., "Boosting Projections to improve surface roughness prediction in high-torque milling operations", Soft Computing, 16(8), 2012, 1427-1437.

Grzenda M., Bustillo A., Zawistowski P., "A soft computing system using intelligent imputation strategies for roughness prediction in deep drilling", Journal of Intelligent Manufacturing, 23(5), 2012, 1733-1743. 
Grzenda M., Bustillo A., Quintana G.and Ciurana J., "Improvement of surface roughness models for face milling operations through dimensionality reduction", Integrated Computer-Aided Engineering, 19(2), 2012, 179-197.

Kaldos A, Pieper HJ, Wolf E, Krause M. Laser machining in die making-a modern rapid tooling process. J Mater Process Technol 2004 11/30;155-156:1815-1820.

Karazi SM, Issa A, Brabazon D. Comparision of ANN and DoE for the prediction of laser machined micro-channel dimensions. Optics and Lasers in Engineering 2009;47:956-964.

Kumar A, Gupta MC. Laser machining of micro-notches for fatigue life. Optics and Lasers in Engineering 2010; 48(6):690-697.

Mahdavinejad, R.A., Khani, N., Fakhrabadi, M.M.S. "Optimization of milling parameters using artificial neural network and artificial immune system", Journal of Mechanical Science and Technology, 26 ( 12 ), 2012, 4097-4104 .

Pham DT, Dimov SS, Petkov PV. Laser milling of ceramic components. Int J Mach Tools Manuf 2007 3;47(3-4):618-626.

Quintana G., Bustillo A., Ciurana J., "Prediction, monitoring and control of surface roughness in high-torque milling machine operations, International Journal of Computer Integrated Manufacturing, 25(12), 2012, 1129-1138.

Rizvi NH, Apte P. Developments in laser micro-machining techniques. J Mater Process Technol 2002;127(2):206-210.

Samant AN, Dahotre NB. Three-dimensional laser machining of structural ceramics. Journal of Manufacturing Processes 2010;12(1):1-7.

Semaltianos NG, Perrie W, Cheng J, French P, Sharp M, Dearden G, et al. Picosecond laser ablation of nickel-based superalloy C263. Applied Physics A: Materials Science and Processing 2010;98(2):345-355.

Yousef BF, Knopf GK, Bordatchev EV, Nikumb SK. Neural network modeling and analysis of the material removal process during laser machining. Int J Adv Manuf Technol 2003;22(1-2):41-53. 